\title{
Paroxetina, placebo y psicoterapia, en la distimia y la depresión menor en adultos mayores
}

Treatment of Dysthimia and Minor Depression in Primary Care. A Randomized Controlled Trial in Older Adults. Williams J., Barret J., Oxman T.et al., JAMA.2000 Sept 27;284, No.12:1519-26.

\section{Objetivo}

Comparar la efectividad de la farmacoterapia y de la psicoterapia en pacientes adultos mayores con depresión menor o distimia en centros de atención primaria.

\section{Diseño}

Ensayo clínico randomizado controlado con placebo.

\section{Lugar}

Cuatro centros de atención primaria de los Estados Unidos, diferentes desde el punto de vista demográfico.

\section{Pacientes}

Participaron 415 pacientes con un promedio de edad de 71 años divididos en dos grupos: quienes padecían depresión menor $(n=204)$ y aquellos con distimia $(n=211)$, con un score en la escala de Hamilton de por lo menos 10.Un total de 311 (74.9\%) completó el estudio.El estudio se desarrolló entre Noviembre de 1995 y Agosto de 1998.

\section{Intervención}

Se aleatorizó* a los pacientes para recibir paroxetina $(n=137)$ comenzando con $10 \mathrm{mg}$./día llegando a una dosis máxima de $40 \mathrm{mg}$./día, placebo $(n=140)$ o una psicoterapia denominada "tratamiento para resolución de problemas en la atención primaria". Para los grupos de placebo y paroxetina se realizaban seis visitas que incluían control de los efectos adversos a la medicación y contención, mientras que el tercer grupo recibía tratamiento psicoterapéutico.

\section{Medición de resultados principales}

Los síntomas depresivos se evaluaron por medio de la escala de depresión de Hamilton y por la escala de Hopkins.

\section{Resultados}

Los pacientes tratados con paroxetina mostraron una mayor resolución de los síntomas comparados con los pacientes que recibieron placebo $(P=.004)$. Los tratados con psicoterapia no evidenciaron una mejoría superior a aquellos bajo placebo $(P=.13)$, pero sus síntomas mejoraron más rápidamente que dicho grupo durante las últimas semanas de tratamiento $(P=.01)$. En los pacientes con distimia la paroxetina mejoró el funcionamiento mental en comparación al placebo en los pacientes que presentaban un funcionamiento intermedio $(\mathrm{P}=.03) \mathrm{o}$ alto $(\mathrm{P}=.01)$ en la línea basal. En cambio, el funcionamiento mental en pacientes con distimia no se vio beneficiado por la psicoterapia comparando con el grupo placebo. La psicoterapia y la paroxetina mejoraron el funcionamiento mental del grupo de pacientes con depresión menor que presentaban un nivel de funcionamiento bajo.

\section{Conclusiones}

La paroxetina demostró ser moderadamente eficaz en el tratamiento de síntomas depresivos y en mejorar el funcionamiento mental de pacientes adultos mayores con distimia y con depresión menor. Los beneficios de la psicoterapia fueron menores, de inicio más lento y mostraron una mayor dependencia a la variación entre centros que los del antidepresivo.

\section{Comentario}

Uno de los méritos de este estudio es poner el foco en los pacientes mayores que padecen distimias o depresiones menores, ya que este grupo está poco considerado en el campo de la investigación, tanto farmacológica como de la psicoterapia 1. Este trabajo muestra que la paroxetina, un antidepresivo IRS, es efectivo en desórdenes afectivos menos severos que las depresiones mayores en pacientes ancianos. Es muy importante la detección y el tratamiento de estos cuadros ya que librados a su evolución natural pueden sufrir severas complicaciones que incluyen el suicidio en un 10 a un $20 \%$ de los casos 2 . La respuesta de los pacientes a la psicoterapia es controvertida; si bien no mostró una diferencia consistente con placebo, presentó una gran variabilidad entre sitios, siendo muy eficaz en uno de los cuatro centros, en el cual el coordinador era el psicoterapeuta con mayor experiencia de todo el ensayo. Este hallazgo conduce a reflexionar sobre lo importante, difícil y complicado que es el entrenamiento en psicoterapia y cómo influye en el tratamiento de los pacientes.

El "tratamiento para la resolución de problemas en la atención primaria" es un modelo de psicoterapia de orientación cognitiva llevado adelante por médicos de atención primaria o por enfermeros/as. Entre las ventajas que presenta podemos mencionar su corta duración (6 sesiones) y el hecho de que representa una opción válida para aquellos pacientes ancianos que por diferentes motivos tienen contraindicada la medicación antidepresiva 3 .

Debido a que no ha sido utilizada en nuestro medio hasta ahora no podemos valorar la respuesta de la población local frente a este método. De todos modos cualquier aporte innovador que beneficie el avance del conocimiento y la mejora de la asistencia a la población anciana amerita nuestra atención y nos estimula a pensar en la posibilidad de investigaciones futuras que permitan validar este método psicoterapéutico en nuestro país.

\section{Dr. Daniel Matusevich [ Servicio de Psiquiatría.Hospital Italiano de Buenos Aires ]}

Referencias

1 Kivela L, Viramo P, Pahkala K.Factors predicting chronicity of depression in elderly primary care patients. International Psychogeriatrics.2000;june, 12;2:183-194. 2 Cattell H.Suicide in the elderly.Advances in psychiatric treatment.2000;6:102-108.

3 Mynors-Wallis L, Gath D, Day A, Baker F. Randomised controlled trial of problem solving treatment, antidepressant medication, and combined treatment for mayor depression in primary care.British Medical Journal.2000;january;320:26-30 